\title{
Willkommen im Elfenbeinturm!
}

\author{
Susanne Robra-Bissantz $\cdot$ Susanne Strahringer
}

(C) Der/die Autor(en) 2020

Unsere Zeitschrift HMD - Praxis der Wirtschaftsinformatik verschreibt sich schon seit ihrer Gründung dem Transfer wissenschaftlicher Erkenntnisse in die Praxis der Wirtschaftsinformatik. Denn, so ist es auf ihrer Website zu lesen, sie möchte, dass ihre Leser*innen aus Hochschulen erfahren, welche Themen in der Praxis ihres Faches Herausforderungen darstellen und aktuell in der Forschung diskutiert werden, während sie IT-Fach- und Führungskräften Lösungsideen und Umsetzungsmöglichkeiten für ihre aktuellen Herausforderungen bietet.

Dem entsprechend ist unser Herausgeberkreis immer mit Mitgliedern aus Forschung und Praxis besetzt und auch in jeder einzelnen Ausgabe achten wir darauf, dass wir Beiträge aus beiden Bereichen präsentieren, die jeweils sowohl für Forscher*innen als auch für Mitarbeiter*innen in der betrieblichen IT-Praxis interessant sind.

Doch was uns in der Wirtschaftsinformatik immer fast natürlich erschien, nämlich, dass Forschung auch etwas für die Praxis tut, ist zunehmend in die wissenschaftliche Diskussion geraten. Ist es wissenschaftlich, wenn man als Forscher*in praxisrelevante Probleme löst? Ist es relevante Forschung, wenn sie im Wesentlichen auf wissenschaftliche Erkenntnis zielt? Wie ist Auftragsforschung für ein Unternehmen wissenschaftlich zu beurteilen? Und: muss sich der Forschende entscheiden, ob er als seriöser Wissenschaftler gelten oder Probleme der Praxis lösen will?

Wir haben das im Herausgeberkreis diskutiert. Und uns ist aufgefallen, dass gerade die Wirtschaftsinformatik immer mehr Forschungsmethoden diskutiert, die

\footnotetext{
S. Robra-Bissantz $(\bowtie)$

Technische Universität Braunschweig, Braunschweig, Deutschland

E-Mail: s.robra-bissantz@tu-braunschweig.de

S. Strahringer

Technische Universität Dresden, Dresden, Deutschland

E-Mail: susanne.strahringer@tu-dresden.de
} 
wissenschaftliche Forschung mit wertvollen Ergebnissen für die Praxis verbindet: Forschung mit der und für die Praxis. Diese Erkenntnis hat uns dazu motiviert, für Sie in der vorliegenden Ausgabe Beiträge zusammenzustellen, die aufzeigen, wie Forscher*innen solche Forschungsmethoden nutzen und damit sowohl anderen Forscher*innen als auch der Praxis aufzeigen, wie es gelingen kann, wissenschaftlich wertvolle Erkenntnis mit praktischer Relevanz zu verbinden. Aber damit nicht genug: In den Beiträgen unserer Autor*innen sind so viele Impulse für gelungene Wissenschaft-Praxis-Kollaborationen in der Forschung zu finden, dass wir uns entschieden haben, den nun aufgenommenen Diskurs dazu nicht nach einer Ausgabe zu beenden, sondern das Thema als wiederkehrende Rubrik in unsere Zeitschrift aufzunehmen. Auch zukünftig werden in der einen oder anderen Ausgabe der HMD also entsprechende Beiträge erscheinen. Aber nun zunächst zur vorliegenden Ausgabe.

Wir beginnen sie mit einem State of the Art, in dem wir Herausgeberinnen nicht nur einen Überblick über Forschungsmethoden für die und mit der Praxis geben, sondern auch verschiedene Kollaborationsformen aufzeigen, die Forschung mit der Praxis ermöglichen. Bei den Kollaborationsformen greifen wir auf eine Studie unserer Kollegin Petra Schubert (et al.) zurück, die uns so gelungen schien, dass wir ihr kaum etwas hinzuzufügen hatten. Eine der darin vorgestellten Kollaborationsformen wird im darauffolgenden Beitrag von Schubert \& Williams am Beispiel der Universität Koblenz und dem dort praktizierten Ansatz IndustryConnect illustriert. Eine andere auf langfristige Zusammenarbeit ausgerichtete Kollaborationsform mehrerer Universitäten mit der Praxis stellen Kammler et al. am Beispiel von Innovationsnetzwerken als Treiber für Wissenschaft-Praxis-Kooperationen vor.

Die nächsten beiden Beiträge zeigen auf, wie Wissenschaftler ihre Forschung im Praxisumfeld durchführen können, auch wenn sie sich mit schwierig zu erfassenden Phänomenen befasst, oder die Meinung von zukünftigen Nutzern zu bislang noch nicht in der Realität zu findenden technologischen Lösungen erhoben werden muss. Es handelt sich dabei um die Beiträge von Fischer \& Riedl zur Messung von digitalem Stress im organisationalen Umfeld sowie Ruess \& Wingartz zu Virtual Reality als Instrument zur Gewinnung von Nutzerfeedback zu Technologieszenarien am Beispiel urbaner Mobilität. Im nächsten Beitrag ist ein erster Schritt dahin getan, die Herausforderungen der Praxis als Ausgangspunkt der Forschung zu sehen. In Arbeitskreise im IT- und Prozessmanagement fokussieren Schröder et al. dabei eine besondere Form der problemorientierten Vermittlung von neuen Erkenntnissen. Scheplitz et al. zeigen in ihrer Forschung in Digitalen Innovationsprojekten umgekehrt auf, wie wesentliches Wissen aus theoretisch fundierten Innovationsprojekten in der Praxis auch in die Wissensbasis zur Forschung zurückfließen kann. Weiter in Richtung einer Kollaboration der Partner geht der Beitrag von Becker et al. zu Open KMU: Mit Action Design Research und Design Thinking gemeinsam innovieren, der eine relativ neue Forschungsmethode, das Action Design Research, in einer Form vorstellt, die mit besonderen Methoden versucht, Herausforderungen der Praxis auf den Grund zu gehen und diese gemeinsam mit den Forschenden zu lösen. Im Beitrag Living Lab: Forschung und Praxis am Universitätscampus stellt Abel eine andere Form der Co-Kreation von Artefakten durch Forscher gemeinsam mit Nutzern - hier am Beispiel einer Plattform zur partizipativen Lebensraumgestaltung vor. 
Die den Schwerpunkt des Heftes abschließenden Beiträge befassen sich mit einem in der Wirtschaftsinformatik wichtigen Artefakt, so genannten Referenzmodellen, die aufgrund ihres angestrebten Allgemeingültigkeitsanspruchs per se geeignet sind, in langfristigen und größeren Konsortialprojekten entwickelt und evaluiert zu werden. Dabei fokussieren Pentek \& Legner in ihrem Beitrag Konsortialforschung zur Entwicklung von Referenzmodellen für die Digitalisierung von Unternehmen industrieübergreifend das Themenfeld des Datenmanagements und Czarnecki \& Dietze mit Gestaltungsorientierte Forschung am Beispiel der Referenzmodellierung die Telekommunikationsindustrie.

Alle Autor*innen des Schwerpunktes teilen in den vorgestellten Beiträgen ihre Erfahrungen und reflektieren Vor- und Nachteile der verwendeten Ansätze. Das soll Sie, liebe Leser*innen, dazu anregen - sei es von Seiten der Praxis oder der Forschung - an solchen Projekten mitzuwirken oder sie gar voranzutreiben, dabei aber mit den richtigen Erwartungen zu starten und auf der Strecke vielleicht auf die eine oder andere in diesem Heft vorgestellte Erkenntnis zurückzugreifen. Von allen, die bereits eigene Erfahrungen gemacht haben und darüber berichten wollen, wünschen wir uns zukünftige Einreichungen zu unserer neuen Rubrik „Forschung für die Praxis“. Wir sind überzeugt, dass der Diskussionsstoff zu diesem Thema noch lange nicht ausgeschöpft ist.

\section{Susanne Robra-Bissantz}

Susanne Strahringer

Funding Open Access funding provided by Projekt DEAL.

Open Access Dieser Artikel wird unter der Creative Commons Namensnennung 4.0 International Lizenz veröffentlicht, welche die Nutzung, Vervielfältigung, Bearbeitung, Verbreitung und Wiedergabe in jeglichem Medium und Format erlaubt, sofern Sie den/die ursprünglichen Autor(en) und die Quelle ordnungsgemäß nennen, einen Link zur Creative Commons Lizenz beifügen und angeben, ob Änderungen vorgenommen wurden.

Die in diesem Artikel enthaltenen Bilder und sonstiges Drittmaterial unterliegen ebenfalls der genannten Creative Commons Lizenz, sofern sich aus der Abbildungslegende nichts anderes ergibt. Sofern das betreffende Material nicht unter der genannten Creative Commons Lizenz steht und die betreffende Handlung nicht nach gesetzlichen Vorschriften erlaubt ist, ist für die oben aufgeführten Weiterverwendungen des Materials die Einwilligung des jeweiligen Rechteinhabers einzuholen.

Weitere Details zur Lizenz entnehmen Sie bitte der Lizenzinformation auf http://creativecommons.org/ licenses/by/4.0/deed.de. 